\title{
BMJ Open Evaluating level of adherence to nicotine replacement therapy and its impact on smoking cessation: a protocol for systematic review and meta-analysis
}

\author{
Amanual Getnet Mersha (D) , ${ }^{1}$ Parivash Eftekhari, ${ }^{1,2}$ Michelle Bovill, ${ }^{1,2}$ \\ Daniel Nigusse Tollosa, ${ }^{1}$ Gillian Sandra Gould (1) ${ }^{1,2}$
}

To cite: Mersha AG, Eftekhari P, Bovill M, et al. Evaluating level of adherence to nicotine replacement therapy and its impact on smoking cessation: a protocol for systematic review and meta-analysis. BMJ Open 2020;10:e039775. doi:10.1136/ bmjopen-2020-039775

- Prepublication history for this paper is available online. To view these files, please visit the journal online (http://dx.doi org/10.1136/bmjopen-2020039775).

Received 25 April 2020 Revised 10 July 2020 Accepted 31 July 2020

Check for updates

(c) Author(s) (or their employer(s)) 2020. Re-use permitted under CC BY-NC. No commercial re-use. See rights and permissions. Published by BMJ.

${ }^{1}$ School of Medicine and Public Health, The University of Newcastle, University Drive, Callaghan, Newcastle 2308, New South Wales, Australia ${ }^{2}$ Hunter Medical Research Institute, Lot 1, Kookaburra Circuit, New Lambton Heights, Newcastle 2305, NSW, Australia

Correspondence to Dr Amanual Getnet Mersha; AmanualGetnet.Mersha@uon. edu.au

\section{ABSTRACT}

Introduction Nicotine replacement therapy (NRT) has proven effective for smoking cessation in clinical trials, however it was found less effective in population-based studies, potentially due to inconsistent or incorrect use of NRT. The aim of this paper is to describe a systematic review protocol to evaluate level of adherence to NRT; the discrepancy of adherence to NRT in clinical and population-based studies and degree of association between level of adherence and success of smoking cessation.

Methods and analysis Literature search will use five databases (Medline, Scopus, Embase, CINAHL and PsycINF0). Studies will be appraised for methodological quality using National Institutes of Health Quality Assessment Tool. To reduce heterogeneity, we will analyse clinical trials and population-based studies separately; pooled analyses will be done among studies that used similar measurements. Heterogeneity of studies will be assessed by Higgins' $\left.\right|^{2}$ statistical test. When studies are adequately homogeneous, results will be pooled using random-effects model with proportion and $\mathrm{ORs}$ with $95 \% \mathrm{Cls}$ and $p$ values for each outcome. We will explain sources of heterogeneity by subgroup analysis or sensitivity analysis. Funnel plots and Egger's regression asymmetry test with $p<0.05$ will be used as a cut-off point to affirm presence of statistically significant publication bias. Statistical analyses will be carried out using Stata V.16 software. Only studies reporting a valid strategy to control for reverse causality will be included.

Discussion This review will provide evidence to support the importance of adherence on rate of smoking cessation and level of adherence to NRT. The findings will be used to inform smoking cessation interventions, researchers and policymakers.

Ethics and dissemination As a systematic literature review, this protocol does not require ethics approval. Research outcomes will be presented at relevant conferences and findings will be published in a relevant peer-reviewed journal.

PROSPERO registration number CRD42020176749.

\section{BACKGROUND}

Globally, 6 million people die each year due to complications of tobacco smoking and another half a million deaths occur as
Strengths and limitations of this study

- First systematic review and meta-analysis protocol to assess the discrepancy in the level of adherence to nicotine replacement therapy (NRT) within and outside of clinical trials, and how the adherence to NRT influences smoking cessation.

- Results may guide researchers and policymakers in the development of strategies to enhance the rate of adherence to smoking cessation medication, if relevant.

- High heterogeneity between studies is likely from a lack of consistency in the definition of adherence and successful smoking cessation.

- To reduce heterogeneity, we will conduct separate meta-analyses of clinical trials and populationbased studies.

a result of secondhand smoke. ${ }^{1}$ Smoking is attributed to $11.5 \%$ of deaths worldwide with half of these deaths occurring in China, Russia, India and the USA. Cardiovascular diseases, various types of cancer and chronic respiratory illnesses have been found to be the most common causes of death associated with tobacco smoking. ${ }^{2}$ At the end of 2015, globally, one in four men and $5.4 \%$ of women smoked tobacco daily, representing a global reduction of tobacco smoking by $28.4 \%$ and $34.4 \%$, respectively since $1990 .^{2}$

Smoking cessation typically encompasses an intention to stop smoking any more cigarettes from a specified time. This attempt is followed by self-conscious struggling against urges to smoke resulting in quitting or continued smoking. The Society for Research on Nicotine and Tobacco recommended clinical trials report prolonged abstinence of at least 6 months and point prevalence as a preferred measure of success of smoking cessation. ${ }^{3}$ The Russell Standard is used to describe self-reported smoking abstinence in 
clinical trials. The guidelines propose continuous abstinence to be measured in 6-month or 12-month periods, from a nominated date. The Russell Standard defines smoking abstinence as no more than five cigarettes from the start of the abstinence period confirmed by a negative biochemical test at the final follow-up. ${ }^{4}$ Smoking cessation often requires behavioural support from a trained tobacco treatment specialist and/or pharmacological therapy. ${ }^{5}$ Pharmacotherapy aims to reduce the extent of withdrawal symptoms and probably works by reducing occurrence of both frequency and strength of urges to smoke. Currently, there are three approved pharmacotherapies widely licensed throughout the globe for smoking cessation: nicotine replacement therapy (NRT), bupropion and varenicline. ${ }^{6}$

Among the aforementioned smoking cessation treatments, NRT is the agent most widely available and frequently used by smokers. ${ }^{7}$ NRT was initially available as transdermal patches and now includes chewing gum, lozenges, oral mist and inhalators. Absorption of nicotine content of these products into the bloodstream via either mucosa or skin replaces the nicotine body receives from tobacco smoking. Replacing nicotine helps with withdrawal symptoms from nicotine and the cravings associated with smoking cessation. ${ }^{8}$ Also, the slower release of nicotine in NRT downregulates nicotinic acetylcholine receptors in the central nervous system. Hence, it will reduce the rewarding effect of smoking and ease smoking cessation. $^{910}$

In a Cochrane review, smoking cessation rates were higher using NRT combination therapy (31.5\%) compared with single NRT therapy $(17.6 \%)$ or bupropion $(19.1 \%)$. However, the review did not find a statistically significant difference in rate of quitting among individuals who used NRT combination therapy and varenicline. ${ }^{11}$ In clinical trials, a higher rate of successful quitting is observed among individuals with greater adherence to smoking-cessation medications. ${ }^{12}$ However, evidence shows half of the people used NRT at a lower dose and for less period of time than optimal suggested by evidence. $^{10}$

Moreover, excluding those who had successfully quit smoking, the rate of prescription refill for NRT was much less than $20 \%$ within a 12 -month follow-up period. ${ }^{13}$ Although NRT is available over the counter, affordability of the cost of NRT was reported to be one of the reasons for early discontinuation in studies conducted in the USA. ${ }^{1014}$

On the other hand, population-based studies have shown that effectiveness of NRT was considerably less when outside of clinical trials. ${ }^{15}$ This may be due to inadequate consumption of pharmacotherapies, ${ }^{16}$ potentially due to a misperception about its safety and effectiveness. ${ }^{17}$ Most people who smoke fail to take smoking cessationsupport medications as prescribed and usually take less than the recommended dose. One factor that has been shown to have a direct effect on the success of smokingcessation treatment is adherence to pharmacotherapy. ${ }^{18}$
Consistent use of NRT has been shown to have a positive impact on the success of smoking cessation, for example, using more pieces of nicotine gum and nicotine lozenges was found to increase quit rates. ${ }^{19} 20$

Medication adherence (defined as 'the extent to which the patient follows medical instructions') comprises numerous health-related behaviours that extend beyond taking prescribed pharmaceuticals. Medical adherence is the main element of the effectiveness of treatment because poor adherence diminishes optimum clinical benefit. ${ }^{21}$ Shiffman et al assessed the effect of consistent daily nicotine patch utilisation and concluded that daily patch use in the first 3 weeks tripled quitting rates at 6 weeks when compared with less compliant use. ${ }^{22}$ Relapse to smoking is an important potential confounder, in which case non-adherence is not the cause but the consequence of relapse. There are two ways to control for this bias: (1) establishing the sequence of non-adherence and relapse and (2) assessing adherence during a prespecified treatment period and determine abstinence only in those who had been continuously abstinent throughout this specified period. A 2019 Cochrane review including five randomised controlled trials for subgroup analysis to assess abstinence demonstrated that interventions directed to increase adherence to medications for tobacco dependence (NRT, bupropion, nortriptyline and varenicline) may increase the rate of short-term and longterm smoking cessation. ${ }^{23}$

A systematic review conducted in 2013 by Raupach et al reported the presence of a modest correlation between adherence to NRT and smoking cessation. ${ }^{12}$ It was difficult to synthesise a pooled result in the review due to few included studies and variations in the definition of adherence and outcome measures. Furthermore, most of the studies included in the review enrolled people who smoked tobacco with additional health behaviour such as alcohol use disorder that limited its reproducibility to the general population. ${ }^{12}$

Despite a well-known link between adherence and clinical outcomes in studies conducted on different medical conditions, ${ }^{24}$ there is no single meta-analysis of adherence and smoking cessation. Most reviews focused on smoking cessation outcomes, rather than on a detailed description of the extent of adherence and its impact on abstinence.

Thus, the current review will have significant importance in terms of presenting further evidence in understanding the association between adherence and abstinence. This pooled data will inform clinicians, researchers and policymakers on the significance of adherence to medications in smoking-cessation therapy.

\section{Specific research questions}

1. What is the existing level of adherence to NRT within clinical trials and outside of trials?

2. What is the extent of discrepancy in the level of adherence to NRT in clinical trials and outside of trials?

3. Is there an association between adherence to NRT and success rate of smoking cessation? 
Table 1 Inclusion and exclusion criteria for study selection

\begin{tabular}{|c|c|c|}
\hline $\begin{array}{l}\text { PICOS } \\
\text { framework }\end{array}$ & Inclusion criteria & Exclusion criteria \\
\hline Population & $\begin{array}{l}\text { Participants will be individuals using NRT for the } \\
\text { purpose of smoking cessation. We will include } \\
\text { studies examining general male and female adult } \\
\text { population ( } 18 \text { years and older), including pregnant } \\
\text { women. We will include relevant studies all over the } \\
\text { world. }\end{array}$ & $\begin{array}{l}\text { Studies restricted to participants with mental } \\
\text { illness and those assessed individuals with other } \\
\text { substance-use disorders, as it may cause significant } \\
\text { clinical heterogeneity to include studies enrolled } \\
\text { only individuals with mental illnesses. For instance, } \\
\text { the level of adherence to NRT was twofold higher } \\
\text { among participants without depressive symptoms as } \\
\text { compared with individuals with depression. }\end{array}$ \\
\hline Intervention & $\begin{array}{l}\text { The intervention can be different treatment durations } \\
\text { of single or combined NRT products taken in forms of } \\
\text { gum, transdermal patch, nasal spray, lozenges or oral } \\
\text { inhalator. }\end{array}$ & $\begin{array}{l}\text { NRT in combination with other smoking cessation } \\
\text { medications such as bupropion and varenicline }\end{array}$ \\
\hline Comparator & $\begin{array}{l}\text { If a clinical trial, the control may be either standard } \\
\text { care or placebo, behavioural intervention or no } \\
\text { intervention. For observational studies comparator is } \\
\text { not applicable. }\end{array}$ & $\begin{array}{l}\text { Comparing NRT with other smoking cessation } \\
\text { medications }\end{array}$ \\
\hline Outcome & $\begin{array}{l}\text { Level of adherence to NRT and/or the impact of } \\
\text { adherence to NRT on rate of successful smoking } \\
\text { cessation. Studies will be included if they address } \\
\text { both level and impact of adherence or either one of } \\
\text { the two outcomes. }\end{array}$ & $\begin{array}{l}\text { No outcomes relating to level of adherence or impact o } \\
\text { adherence on smoking outcomes }\end{array}$ \\
\hline Study design & $\begin{array}{l}\text { All study designs which used a quantitative } \\
\text { methodology including case-control, cohort, cross- } \\
\text { sectional, longitudinal, randomised control trials and } \\
\text { others. No limitation on publication date, sample size, } \\
\text { setting, follow-up period. } \\
\text { Only studies reporting a valid strategy to control for } \\
\text { relapse will be included in the review. }\end{array}$ & $\begin{array}{l}\text { Commentaries, expert opinion, abstracts, conference } \\
\text { presentation without complete results }\end{array}$ \\
\hline
\end{tabular}

NRT, nicotine replacement therapy; PICOS, Population, Intervention, Comparison, Outcome and Study.

\section{METHODS}

This protocol has been developed following the Preferred Reporting Items for Systematic Reviews and Meta-Analyses for Protocols (PRISMA-P) guidelines ${ }^{25}$ (online supplemental file 1). To present the findings of our review, we will use the PRISMA ${ }^{26}$ and Meta-analysis Of Observational Studies in Epidemiology guidelines for meta-analyses of observational studies. ${ }^{27}$

\section{Inclusion and exclusion criteria}

Eligibility criteria are illustrated in table 1 using the Population, Intervention, Comparison, Outcome and Study strategy. ${ }^{28}$

\section{Search strategy}

Literature search will be conducted from five databases: Medline, Scopus, Embase, CINAHL and PsycINFO. Additionally, we will check reference lists of included articles. A practical tool for searching health-related grey literature ${ }^{29}$ will be used to search for ongoing and unpublished reports. The following websites and databases will be searched: Foundation for a Smoke-free World, Tobacco. org, Centres for Disease Control and Prevention Smoking and Health Resource Library, US National Comprehensive Cancer Network, National Institute for Health and
Care Excellence, Quitline Consortium website and the Ottawa Heart Institute's Ottawa Model for Smoking Cessation. The search strategy was developed with the assistance of an experienced librarian. The search will span from the start of indexing to 25 February 2020 and citation alerts created. The free-text words (with truncation) and Medical Subject Headings terms will be combined using Boolean logic operators: AND, OR and NOT. A combination of keywords and phrases like: Smoking, "Smoking cessation", Cessation, Smoke, Cigarette, Quitting, "Quitting Smoking", "Medication Adherence", Adherence, Discontinuation, Compliance, Non-Compliance, Nonadherence, "Treatment Compliance", "Therapeutic Compliance", "Nicotine replacement therapy", NRT, "Nicotine patch", Patch, "Nicotine gum", "Nicotine inhaler", Inhaler, Lozenge, "Nicotine spray", Pharmacotherapies, "Drug therapies", "Pharmacological therapy" and "Medication treatment" will be used to search articles in the databases. Citations will be gathered using Endnote reference management software V.9 and will be exported to Covidence for screening (online supplemental file 2).

\section{Identification and selection of studies}

Two authors (AGM, DNT) will screen the titles and abstracts of each article independently using Covidence ${ }^{30}$ 
by applying a customised inclusion/exclusion criteria. Two authors (AGM, DNT) will independently review the full text. Final inclusion of the studies will be determined by agreement of both reviewers and when there is disagreement, a third author will adjudicate the decision.

\section{Data extraction}

Data extraction will be performed by two reviewers (AGM, DNT) independently for each article. A data extraction template will be prepared and pretested by extracting data from three articles ${ }^{18} 3132$ and necessary modifications will be made. Further amendments will also be done if necessary. When there is a disagreement in data extraction between the reviewers, it will be resolved through discussion and mutual agreement between the investigators.

The template will have four main sections:

1. Identity of the study: study title; first author; country of the study; publication year.

2. Methodological characteristics of included studies: study design; study objective or research question or hypothesis; sample characteristics (eg, sample size, sex, age, race, groups); stated length of follow-up; inclusion/exclusion criteria; type of NRT used in the study; type of intervention; definition of successful smoking cessation used by each study (eg, self-reported, 7-day point prevalence, continuous abstinence); measure of adherence; validation of smoking cessation (biochemical markers); intention to treat analysis.

3. Main findings: level of adherence to NRT and associations between adherence and successful smoking cessation.

4. Conclusions: key conclusions of the study.

\section{Quality assessment}

National Institutes of Health (NIH) Quality Assessment Tool for observational and interventional studies will be used to assess the quality of studies. ${ }^{33}$ The NIH tools comprised numerous items for each study design that can evaluate potential errors in study methods, study implementation, including sources of bias (eg, patient selection, performance, attrition and detection), confounding, study power, strength of causality in the association between interventions and outcomes, and other factors. Quality reviewers select 'yes,' 'no' or 'cannot determine/ not reported/not applicable' in response to each item on the tool. Two reviewers (AGM, DNT) will independently assess the quality of each study. Discussion regarding differences will follow and consensus will be reached. When agreement cannot be reached, a third reviewer will adjudicate the decision.

\section{Data analysis}

To reduce heterogeneity between studies, we will analyse clinical trials and population-based studies separately. Pooled analyses will be done among studies that used similar measurements to assess level of adherence and successful smoking cessation.
Meta-analyses, subgroup analysis and/or descriptive analysis will be performed based on the included studies. Meta-analysis will be conducted by using Stata software (V.16, Stata Corp LP, College Station, Texas, USA) for those outcomes with enough studies to undertake metaanalysis. ${ }^{34}$ Statistical heterogeneity between studies will be evaluated using the Higgins' $\mathrm{I}^{2}$ statistic. Heterogeneity will be considered low, moderate and high when the values are below $25 \%$, between $25 \%$ and $75 \%$, and above $75 \%$, respectively. ${ }^{35}$ Studies with relatively similar follow-up period and assessment strategy for adherence and smoking cessation will be included in the metaanalysis. A statistical heterogeneity above $75 \%$ using Higgins' $\mathrm{I}^{2}$ statistic will define substantial heterogeneity. Source of heterogeneity will be investigated by employing subgroup analysis or sensitivity analysis and it will be stated as a limitation so that the outcome will be interpreted with caution. When studies are adequately homogeneous, we will pool the results using proportions, ORs and risk differences, and we will calculate $95 \%$ CIs and $p$ values for each outcome. When there are more than one outcomes, we will state all the outcomes in a table and outcome of interest for our systematic review will be pointed out. When studies have multiple timepoint assessments, we will use the longest follow-up period available. The effect estimates from each study will be extracted and used in the meta-analysis. Then the rate of adherence will be compared between real world studies and clinical trials using the overall effect estimate from the meta-analysis. If there is a difference in the level of adherence between the two sub-groups, we will examine whether the difference is statistically significant or not. To evaluate the association between adherence to NRT and abstinence, studies with relatively similar measurement of adherence as well as smoking cessation will be used, and result will be presented with OR with $95 \%$ CI. Each outcome will be combined and calculated according to the statistical guidelines referenced in the Cochrane Handbook for Systematic Reviews. The Mantel-Haenszel method will be used for the fixed effect model if tests of heterogeneity are not significant. If statistical heterogeneity is moderate or high, the random effects model with DerSimonian-Laird will be chosen. If it is difficult to pool results, we will not perform a meta-analysis and a narrative summary will be done. Egger's regression asymmetry test with $p<0.05$ will be used as a cut-off point to affirm presence of statistically significant publication bias. ${ }^{36} \mathrm{~A}$ narrative synthesis of the findings from included studies as well as a summary table will be created to have a more detailed comment on the extracted data.

\section{Analysis of subgroups or subsets}

Subgroup analysis will be conducted based on the type of studies (population based and clinical trials); definitions used to evaluate level of adherence and smoking cessation, based on participant characteristics and others depending on the included articles. We will conduct a pooled analysis among studies that enrolled pregnant 
women separately as pregnant women may use NRT differently because of fetal and neonatal concerns. ${ }^{37}$

\section{Definitions of outcomes \\ Adherence}

The WHO defines adherence as 'the extent to which the patient follows medical instructions ${ }^{38}$ However, in regards with smoking cessation pharmacotherapy there are inconsistencies in the definition of adherence as well as its measurement. We will present definitions used and measurements used to determine adherence to NRT in the studies.

\section{Abstinence}

Abstinence is defined as the proportion of participants who achieved point prevalence up to a given point of time. The assessment of abstinence is usually based on self-report or measuring salivary cotinine or exhaled carbon monoxide. The cut-off values used for each of the above biochemical measures used in each study will be presented.

\section{Patient and public involvement}

As our research will use previously published studies, patient and public involvement are not required.

\section{Ethics and dissemination}

This research will use previously published studies, so it does not require ethical clearance to retrieve already published articles. Research outcomes will be presented at relevant conferences and findings will be published in a relevant peer-reviewed journal.

\section{Implications of the review}

This review will provide a summary of the evidence so that healthcare providers and policymakers can use the findings to understand and improve adherence to NRT and corresponding rates of successful smoking cessation. Moreover, this review will have importance in terms of giving new evidence in understanding the association between adherence and abstinence from smoking. It will direct researchers and policymakers to develop strategies that will enhance rate of adherence to smoking cessation medication if found relevant.

\section{Possible limitations}

Lack of consistency in the definition of adherence, successful smoking cessation and how it is measured may lead to systematic biases and may affect the level of homogeneity. Hence, we will group studies and conduct a pooled analysis among studies that have used relatively similar follow-up period to assess adherence and cessation. Furthermore, we will use random effect model to pool an overall result to account for these differences if level of heterogeneity is high. A strength of this review is it will be the first, to the best of our knowledge, to present pooled data on the impact of adherence to NRT on the success of smoking cessation.
Twitter Amanual Getnet Mersha @MershaGetnet, Parivash Eftekhari @DrPEftekhari, Michelle Bovill @michelle_bovill and Gillian Sandra Gould @GillianSGould

Acknowledgements The authors would like to thank Ms Debbie Booth, a senior librarian at the university of Newcastle, for helping develop the search strategies for the review.

Contributors AGM, GSG, PE and MB contributed to the conception of the study. The manuscript protocol was drafted by AGM and was revised by GSG, PE and MB. The search strategy was developed by AGM and PE. AGM and DNT will be involved in extracting data of included studies, assess the risk of bias and complete the data synthesis. GSG will adjudicate the disagreements, ensure that no errors are introduced during the study, and oversee the study. All authors approved the publication of the protocol.

Funding The authors have not declared a specific grant for this research from any funding agency in the public, commercial or not-for-profit sectors.

Competing interests None declared.

Patient and public involvement Patients and/or the public were not involved in the design, or conduct, or reporting, or dissemination plans of this research.

Patient consent for publication Not required.

Provenance and peer review Not commissioned; externally peer reviewed.

Open access This is an open access article distributed in accordance with the Creative Commons Attribution Non Commercial (CC BY-NC 4.0) license, which permits others to distribute, remix, adapt, build upon this work non-commercially, and license their derivative works on different terms, provided the original work is properly cited, appropriate credit is given, any changes made indicated, and the use is non-commercial. See: http://creativecommons.org/licenses/by-nc/4.0/.

ORCID iDs

Amanual Getnet Mersha http://orcid.org/0000-0002-1183-8517

Gillian Sandra Gould http://orcid.org/0000-0001-8489-2576

\section{REFERENCES}

1 World Health Organization, GBD 2015 Tobacco Collaborators. WHO global report on trends in prevalence of tobacco smoking 2015, 2015. Available: https://apps.who.int/iris/handle/10665/156262 2015

2 GBD 2015 Tobacco Collaborators. Smoking prevalence and attributable disease burden in 195 countries and territories, 19902015: a systematic analysis from the global burden of disease study 2015. Lancet 2017;389:1885-906.

3 Hughes JR, Keely JP, Niaura RS, et al. Measures of abstinence in clinical trials: issues and recommendations. Nicotine Tob Res 2003;5:13-26.

4 West R, Hajek P, Stead L, et al. Outcome criteria in smoking cessation trials: proposal for a common standard. Addiction 2005;100:299-303.

5 West R. Tobacco smoking: health impact, prevalence, correlates and interventions. Psychol Health 2017;32:1018-36.

6 Wadgave U, L N, Nagesh L. Nicotine Replacement Therapy : An Overview. Int J Health Sci 2016;10:407-16.

7 Shiffman S, Brockwell SE, Pillitteri JL, et al. Use of smokingcessation treatments in the United States. Am J Prev Med 2008;34:102-11.

8 Lindson N, Aveyard P. An updated meta-analysis of nicotine preloading for smoking cessation: investigating mediators of the effect. Psychopharmacology 2011;214:579-92.

9 Benowitz NL. Nicotine addiction. N Engl J Med 2010;362:2295-303.

10 Burns EK, Levinson AH. Discontinuation of nicotine replacement therapy among smoking-cessation attempters. Am J Prev Med 2008;34:212-5.

11 Cahill K, Stevens S, Perera R, et al. Pharmacological interventions for smoking cessation: an overview and network meta-analysis. Cochrane Database Syst Rev 2013;31:Cd009329.

12 Raupach T, Brown J, Herbec A, et al. A systematic review of studies assessing the association between adherence to smoking cessation medication and treatment success. Addiction 2014;109:35-43.

13 Solberg LI, Parker ED, Foldes SS, et al. Disparities in tobacco cessation medication orders and fills among special populations. Nicotine Tob Res 2010;12:144-51.

14 Yingst JM, Veldheer S, Hrabovsky S, et al. Reasons for nonadherence to nicotine patch therapy during the first month of a quit attempt. Int J Clin Pract 2015;69:883-8. 
15 Alberg AJ, Patnaik JL, May JW, et al. Nicotine replacement therapy use among a cohort of smokers. J Addict Dis 2005;24:101-13.

16 Johnson RE, Stevens VJ, Hollis JF, et al. Nicotine chewing gum use in the outpatient care setting. J Fam Pract 1992;34:61-5.

17 Coleman T, Chamberlain C, Cooper S, et al. Efficacy and safety of nicotine replacement therapy for smoking cessation in pregnancy: systematic review and meta-analysis. Addiction 2011;106:52-61.

$18 \mathrm{Lam} \mathrm{T-H}$, Abdullah ASM, Chan SSC, et al. Adherence to nicotine replacement therapy versus quitting smoking among Chinese smokers: a preliminary investigation. Psychopharmacology 2005;177:400-8.

19 Shiffman S, Rolf CN, Hellebusch SJ, et al. Real-World efficacy of prescription and over-the-counter nicotine replacement therapy. Addiction 2002;97:505-16.

20 Shiffman S. Use of more nicotine lozenges leads to better success in quitting smoking. Addiction 2007;102:809-14.

21 Schneller-Pavelescu L, Vergara-de Caso E, Martorell A, et al. Interruption of oral clindamycin plus rifampicin therapy in patients with hidradenitis suppurativa: an observational study to assess prevalence and causes. J Am Acad Dermatol 2019;80:1455-7.

22 Shiffman S, Sweeney CT, Ferguson SG, et al. Relationship between adherence to daily nicotine patch use and treatment efficacy: secondary analysis of a 10-week randomized, double-blind, placebocontrolled clinical trial simulating over-the-counter use in adult smokers. Clin Ther 2008;30:1852-8.

23 Hollands GJ, Naughton F, Farley A, et al. Interventions to increase adherence to medications for tobacco dependence. Cochrane Database Syst Rev 2019;8:Cd009164.

24 DiMatteo MR, Giordani PJ, Lepper HS, et al. Patient adherence and medical treatment outcomes: a meta-analysis. Med Care 2002;40:794-811.

25 Moher D, Shamseer L, Clarke M, et al. Preferred reporting items for systematic review and meta-analysis protocols (PRISMA-P) 2015 statement. Syst Rev 2015;4:1.

26 Moher D, Liberati A, Tetzlaff J, et al. Preferred reporting items for systematic reviews and meta-analyses: the PRISMA statement. $J$ Clin Epidemiol 2009;62:1006-12.
27 Stroup DF, Morton SC, Berlin JA, et al. Meta-Analysis of observational studies in epidemiology: a proposal for reporting. Jama 2000;283:2008-12.

28 Schardt C, Adams MB, Owens T, et al. Utilization of the PICO framework to improve searching PubMed for clinical questions. BMC Med Inform Decis Mak 2007;7:16.

29 Cadth. Grey matters: a practical tool for searching health-related grey literature.Ottawa, 2018. Available: https://www.cadth.ca/ resources/finding-evidence

30 Covidence. Covidence systematic review software, veritas health innovation, Melbourne, Australia. Available: www.covidence.org

31 Hollands GJ, Sutton S, McDermott MS, et al. Adherence to and consumption of nicotine replacement therapy and the relationship with abstinence within a smoking cessation trial in primary care. Nicotine Tob Res 2013:15:1537-44.

32 Schneider M-P, van Melle G, Uldry C, et al. Electronic monitoring of long-term use of the nicotine nasal spray and predictors of success in a smoking cessation program. Nicotine Tob Res 2003;5:719-27.

$33 \mathrm{NIH}$. NIH study quality assessment tools. Available: https:// www.nhlbi.nih.gov/health-topics/study-quality-assessment-tools [Accessed 24 Feb 2020].

34 StataCorp. Stata statistical software: release 16 College Station, tx: StataCorp Lp. Available: https://www.stata.com/new-in-stata/

35 Higgins JPT. Cochrane Handbook fows of interventions. http://www. mri.gov.lk/assets/Uploads/Research/Cochrane-Hand-booktext.pdf.

36 Huedo-Medina TB, Sánchez-Meca J, Marín-Martínez F, et al. Assessing heterogeneity in meta-analysis: Q statistic or 12 index? Psychol Methods 2006;11:193-206.

37 Bar-Zeev Y, Lim LL, Bonevski B, et al. Nicotine replacement therapy for smoking cessation during pregnancy. Med J Aust 2018;208:46-51.

38 World Health Organization. Adherence to long term therapies, 2001. Available: https://www.who.int/chp/knowledge/publications/ adherence report/en/

39 Hood NE, Ferketich AK, Paskett ED, et al. Treatment adherence in a lay health adviser intervention to treat tobacco dependence. Health Educ Res 2013;28:72-82. 